\title{
A Genética nos Cuidados de Saúde Primários: Uma Perspetiva Multidisciplinar
}

\section{Genetics on Primary Healthcare: A Multidisciplinary Perspective}

Sara MAGALHÃES $\rrbracket^{1}$, Milena PANEQUE ${ }^{2,3,4}$, João SILVA ${ }^{2,3,4}$

Acta Med Port 2016 Oct;29(10):581-582 - http://dx.doi.org/10.20344/amp.7947

Palavras-chave: Cuidados de Saúde Primários; Genética Médica; Portugal.

Keywords: Genetics, Medical; Portugal; Primary Health Care.

Foi objetivo deste artigo a sensibilização dos profissionais de saúde para as atuais potencialidades e constrangimentos dos cuidados de saúde primários relativamente à área da genética. Com uma visão multidisciplinar, os autores discutem as necessidades de apoio na área da Genética Médica Clínica (GMC) nos Cuidados de Saúde Primários (CSP), no sentido de uma eventual reestruturação organizacional de prestação de serviços, à semelhança do que acontece noutros países. Os autores refletem ainda, acerca das lacunas formativas existentes e as potencialidades de prevenção que os conhecimentos em genética possibilitam, com os consequentes ganhos, quer para o utente quer os profissionais envolvidos na prestação destes cuidados. A melhoria dos cuidados de saúde tem conduzido a uma diminuição da mortalidade e aumento da morbilidade, consequente ao aumento da esperança média de vida. Temos vindo a assistir à transição da 'era infeciosa' para a 'era das doenças crónicas', perspetivando-se no futuro a interpretação da doença ao nível das suas variantes genéticas. ${ }^{1}$ Os avanços da medicina salientam cada vez mais o papel da genética não apenas na doença mas também a sua aplicação na prevenção e no tratamento. ${ }^{1}$

Sabe-se por exemplo que as doenças cardiovasculares, a diabetes mellitus e o cancro, possuem causa multifatorial. ${ }^{1}$ Permanece, no entanto, difícil de estimar o risco da sua recorrência nas diferentes famílias pela variabilidade do contributo da hereditariedade genética. ${ }^{1}$ Por outro lado, existem doenças com padrões de hereditariedade mendeliana, nas quais é possível determinar a priori o risco de recorrência familiar. ${ }^{1}$

Refere-se como exemplos a doença de MachadoJoseph, com elevada prevalência em Portugal continental $(1 / 100000)^{2}$ e a polineuropatia amiloidótica familiar (PAF), vulgarmente conhecida como doença dos pezinhos e com maior prevalência em municípios como a Póvoa de Varzim/ Vila do Conde, Braga e Barcelos. ${ }^{3}$ Portugal é reconhecido como o maior foco mundial para a doença, estimando-se

a existência de 1657 casos, para uma das doenças atualmente elegíveis para diagnóstico genético pré-implantatório (DGPI). ${ }^{3}$ Outra doença neurológica de início tardio de transmissão autossómica dominante é a doença de Huntington, com uma prevalência nacional estimada superior a 2-5/100 000. ${ }^{4}$ Todas estas doenças com hereditariedade autossómica dominante (AD) possuem um risco de recorrência de $50 \%$ na descendência de indivíduos afetados. Portugal é assim considerado um importante nicho para algumas doenças neurodegenerativas, apresentando por isso necessidades específicas nas áreas da prevenção e aconselhamento genético. ${ }^{3}$ Relativamente às doenças autossómicas recessivas (AR), refere-se a fibrose quística, doença hereditária multissistémica com várias formas de apresentação e que em Portugal tem uma prevalência estimada de 1/2 500 nados-vivos. ${ }^{5}$ No caso das anemias hereditárias, embora se verifique uma baixa prevalência de $\beta$-talassémia $(0,45 \%)$, existem distritos como Évora $(1,57 \%)$ e Beja $(1,11 \%)$, em que esta atinge valores de prevalência mais elevados. ${ }^{6}$

Um estudo Português envolvendo doentes com hemocromatose 7 (doença AR), revelou que $78 \%$ dos participantes respondeu que gostaria de a receber do seu médico de família. Acreditamos que apesar das especificidades desta doença, tal opinião se justifica pelo acompanhamento longitudinal, holístico e abrangente que a Medicina Geral e Familiar presta ao doente e à sua família, sendo nesta que estes depositam mais expectativas na gestão da sua saúde. No entanto, no mesmo estudo, 69\% dos doentes mostrou depositar maior confiança na discussão da sua doença com profissionais de outras especialidades. Este facto denota a necessidade de um conhecimento mais especializado em serviços de proximidade, como os CSP.

A formação dos profissionais dos CSP na área da GMC é ainda maioritariamente limitada à formação básica instituída nas Faculdades. Este facto, aliado à já reconhecida limitada capacidade de resposta da GMC às

1. Unidade de Saúde Familiar Ponte. Agrupamento de Centros de Saúde do Alto Ave. Guimarães. Portugal.

2. Centro de Genética Preditiva e Preventiva. Universidade do Porto. Porto. Portugal.

3. Instituto de Biologia Molecular e Celular. Universidade do Porto. Porto. Portugal.

4. Instituto de Investigação e Inovação em Saúde. Universidade do Porto. Porto. Portugal.

$\square$ Autor correspondente: Sara Magalhães. saraimaga@gmail.com

Recebido: 16 de junho de 2016 - Aceite: 31 de agosto de 2016 | Copyright $\odot$ Ordem dos Médicos 2016 
necessidades do nosso país, ${ }^{6}$ poderá condicionar 0 usufruto das potencialidades que esta disciplina tem vindo a disponibilizar em áreas tão importantes como a oncogenética, a neurologia, o diagnóstico pré-natal e a farmacogenética. ${ }^{1}$ Perante a necessidade de articulação entre os CSP e a GMC, parece premente organizar a prestação destes cuidados numa vertente de proximidade, com garantias de maior acessibilidade. ${ }^{6}$

Dos vários modelos de ensino que têm sido estudados no sentido de melhor capacitar os CSP nesta área, a formação médica contínua é aquela que parece reunir os melhores resultados. ${ }^{8}$ Para além da necessidade de atualização dos CSP, nomeadamente em questões preventivas e com o apoio dos médicos de GMC, considerámos também necessário que se concretize um novo modelo de comunicação entre a Medicina Geral e Familiar e a especialidade de GMC. Um dos modelos atuais, denominado 'modelo em pirâmide'9 baseia-se no conceito de que o conhecimento da GMC, disponibilizado numa organização vertical com uma rede de comunicação privilegiada, poderá representar ganhos nos cuidados prestados à comunidade. Do mesmo modo, a integração de um médico e enfermeiro de família com formação específica na avaliação do risco genético e com acesso a meios de comunicação e informação especializada no seio dos CSP, parece constituir um dos modelos que melhor responde às necessidades futuras..$^{10}$ Um exemplo bem-sucedido deste modelo são os cuidados prestados aos doentes com hemocromatose hereditária nos CSP, na Irlanda. ${ }^{7}$ Este modelo de proximidade, constituiria uma potencial resposta aos números e tempos de espera nesta área de referenciação em Portugal, providenciando um acesso precoce e equitativo das famílias afetadas ou em risco de doença genética, à informação, ao acompanhamento diagnóstico e preventivo, bem como ao aconselhamento genético de que estas deverão beneficiar.

A presente reflexão pretende alertar para os atuais constrangimentos existentes em Portugal no que concerne à organização dos serviços de GMC, nomeadamente em resposta às necessidades crescentes de referenciação sentidas nos CSP. Do mesmo modo salienta as necessidades de atualização dos profissionais dos CSP relativamente à prevenção nesta área, sublinhando as potencialidades de uma atuação conjunta com os centros de GMC na permeabilização da rede de referenciação. A atual prestação de cuidados de saúde em genética clínica é insuficiente e limitada no seu essencial caráter preventivo devido à ausência de um plano de formação contínuo e verticalizado da especialidade de GMC para a Medicina Geral e Familiar. Tais mudanças deverão vocacionar os serviços de GMC para serviços de consultoria, idealmente com atuação no terreno dos CSP.

\section{CONFLITOS DE INTERESSE}

Os autores declaram não terem qualquer conflito de interesse relativamente ao presente artigo.

\section{FONTES DE FINANCIAMENTO}

Os autores declaram não ter recebido subsídios ou bolsas para a elaboração do artigo.

\section{REFERÊNCIAS}

1. Becker F, Becker F, van El CG, Ibarreta D, Zika E, Hogarth S, Borry $P$, et al. Genetic testing and common disorders in a public health framework: how to assess relevance and possibilities. Eur J Hum Genet. 2011;19:S6-44.

2. Bettencourt C, Lima M. Machado-Joseph Disease: from first descriptions to new perspectives. Orphanet J Rare Dis. 2011;2:35.

3. Valdrez K, Alves A, Coelho T, Silva S. Prevalência do uso de diagnóstico genético pré-implantação na Unidade Clínica de Paramiloidose do Centro Hospitalar do Porto. Acta Med Port. 2014;27:710-6.

4. Costa MC, Magalhães $P$, Guimarães L, Maciel P, Sequeiros J, Sousa A. Molecular diagnosis of Huntington disease in Portugal: implications for genetic counselling and clinical practice. Eur J of Hum Genet. 2003;11:872-8.

5. Grangeia A, Alves S, Gonçalves L, Santos A, Barros A, Moura C, et al. Prevalence of CF and spectrum of CFTR mutations in the Portuguese population. J Cyst Fibros. 2015;14:138
6. Direcção Geral de Saúde. Rede de referenciação Hospitalar de Genética Médica. Lisboa: DGS; 2004.

7. Teixeira E, Borlido-Santos J, Brissot P, Butzeck B, Courtois F, Evans $\mathrm{RW}$, et al. The importance of the general practitioner as an information source for patients with hereditary haemochromatosis. Patient Educ Couns. 2014;96:86-92.

8. Paneque M, Turchetti D, Jackson L, Lunt P, Houwink E, Skirton H. A systematic review of interventions to provide genetics education for primary care. BMC Family Practice. 2016;17:89.

9. Julian-Reynier C, Nippert I, Calefato JM, Harris H, Kristoffersson U, Schmidtke J, et al. Genetics in clinical practice: general practitioners' educational priorities in European countries. Genet Med. 2008;10:10713.

10. Battista RN, Blancquaert I, Laberge A, Schendel N, Leduc N. Genetics in health care: An overview of current ande emerging models. Public Health Genomics. 2012;15:34-45. 